\title{
BMJ Open Scoping review protocol: the use of telemedicine in providing opioid agonist treatment and related psychosocial supports
}

\author{
Des Crowley (D) , ${ }^{1}$ Robyn Homeniuk (D) ,2 Ide Delargy ${ }^{3}$
}

To cite: Crowley D, Homeniuk R, Delargy I. Scoping review protocol: the use of telemedicine in providing opioid agonist treatment and related psychosocial supports. BMJ Open 2020;10:e040556. doi:10.1136/ bmjopen-2020-040556

- Prepublication history for this paper is available online. To view these files, please visit the journal online (http://dx.doi. org/10.1136/bmjopen-2020040556).

Received 20 May 2020 Revised 12 0ctober 2020 Accepted 05 November 2020

Check for updates

(c) Author(s) (or their employer(s)) 2020. Re-use permitted under CC BY-NC. No commercial re-use. See rights and permissions. Published by BMJ.

${ }^{1}$ School of Medicine, University College Dublin, Dublin, Ireland ${ }^{2}$ Management of Addiction in Primary Care, Irish College of General Practitioners, Dublin, Ireland

${ }^{3}$ Substance Misuse, Irish College of General Practitioners, Dublin, Ireland

Correspondence to

Dr Des Crowley;

doctordes@hotmail.com

\section{ABSTRACT}

Introduction The global opioid-related disease burden is significant. Opioid agonist treatment (OAT) can be effective in reducing illicit opioid use and fatal overdose, and improving multiple health and social outcomes. Despite evidence for its effectiveness, there are significant deficits in OAT globally. COVID-19 has required rapid adaptation of remote models of healthcare. Telemedicine is not used routinely in OAT, and little is known about the current levels of use and effectiveness. The objective of this review is to describe models of telemedicine and their efficacy. Methods and analysis This scoping review uses the review methodology described by Arksey and 0'Malley and adapted by Levac et al. The search strategy developed by the medical librarian at the Irish College of General Practitioners in conjunction with the research team will involve five databases (PubMed, EMBASE, the Cochrane Library, PsycInfo and OpenGrey) and the hand searching of reference lists. A limited initial search of two databases will be completed to refine search terms, followed by a second comprehensive search using newly refined search terms of all databases and finally hand searching references of included studies. To be included, studies must report on remote ways of providing OAT (including assessment, induction and monitoring) or related psychosocial support; be published in English after 2010. Two researchers will independently screen titles, abstracts and full-text articles considered for inclusion. Data will be extracted onto an agreed template and will undergo a descriptive analysis of the contextual or process-oriented data and simple quantitative analysis using descriptive statistics.

Ethics and dissemination Research ethics approval is not required for this scoping review. The results of this scoping review will inform the development of a national remote model of OAT. The results will be published in peerreviewed journals and presented at relevant conferences.

\section{INTRODUCTION}

Health services globally are adapting to cope with COVID-19. Many services are implementing dynamic and innovative changes to manage the challenges posed by COVID19. ${ }^{1-3}$ Much of these changes have focused on the delivery of remote care by telephone and video.

\section{Strengths and limitations of this study}

This is the first scoping review of remote models of providing opioid agonist treatment (OAT) and related psychosocial support.

- The search will be conducted quickly with results disseminated rapidly to inform how remote care may be useful in managing COVID-19-related risks both in OAT and other healthcare settings.

- The search strategy includes four electronic databases for peer-reviewed literature and one database for grey literature sources.

- Due to date and language restrictions, historical models and those developed in non-Englishspeaking countries will be excluded.

The global opioid-related disease burden is an increasing public health concern. Worldwide, there are an estimated 26.8 million people opioid dependent resulting in over 86000 deaths annually. ${ }^{4}$ People who use opioids (PWUO), in particular, people who inject opioids have increased medical and social needs. ${ }^{45}$ They experience increased levels of homelessness and imprisonment. ${ }^{5}$ There is also an increased prevalence of HIV and hepatitis $\mathrm{C}$ infection among people who inject drugs causing increased rates of morbidity and mortality in this group compared with the general population. ${ }^{6}$

Opioid agonist treatment (OAT) can safely and effectively reduce illicit drug use, the transmission of HIV and hepatitis C infection, and mortality. Furthermore it improves mental and physical well-being. ${ }^{7}$ Engagement and retention in treatment improve outcomes and disruption is associated with increased risk of overdose, particularly postprison release. ${ }^{78}$ There are many existing treatment deficits for PWUO including inadequate access to OAT, needle and syringe programmes, and overdose prevention and naloxone programmes. ${ }^{9}$ These deficits are 
even more concerning during the COVID-19 pandemic and create challenges in relation to compliance with social distancing, self-isolation and cocooning. ${ }^{10}$

Maintaining continuity of care and protecting frontline staff have been identified as priorities during this pandemic and drug treatment services will have similar challenges to other healthcare settings. ${ }^{10}$ Similar to other services, reducing the requirement for face-to-face care is recommended. ${ }^{3}$

To manage the challenges posed by COVID-19, Irish OAT services have widely adapted to telemedicine to conduct risk triaging, assessment, reviews, and for providing counselling and psychosocial support. Patients have responded well to these measures and like other models of remote care have reported satisfaction with virtual consultations due to reduced waiting times and travel costs. ${ }^{11}$ While telephone consultations are suitable for most patients, video linkage may be required for those with greater health and psychosocial needs. ${ }^{2}$ At the time of writing, the Irish health service has yet to agree with a suitable electronic platform and does not recommend the use of mainstream video conferencing services for clinical purposes. Historically small numbers of OAT services have successfully used telemedicine for hepatitis $\mathrm{C}$ treatment and providing psychological and other supports. ${ }^{11-13}$ Mental health services have also reported success with telemedicine, and general practice was one of the first medical specialties to adapt to its use in the management of COVID-19. ${ }^{2} 14$

Expanding and adapting OAT services are critical to minimise the impact of COVID-19 and remote care models provide opportunities. Telemedicine may also have the added benefits of increasing access and improving retention in OAT into the future. ${ }^{11}$ The objectives of this scoping review are to: describe these models (including their implementation, prevalence and utility in overcoming challenges to OAT delivery), report on their efficacy and identify gaps in the literature. When completed the review will inform how OAT services can adapt to manage the immediate and emerging risks of COVID-19 and improve OAT delivery internationally. ${ }^{15}$

\section{METHODS AND ANALYSIS}

\section{Protocol development}

This study will use the five-stage framework for scoping reviews as originally outlined by Arksey and O'Malley and updated by Levac et al.$^{1516}$ The five stages in this framework are: (1) identifying the research question, (2) identifying relevant studies, (3) study selection, (4) charting the data, and (5) collating, summarising and reporting. The review will be reported according to Preferred Reporting Items for Systematic reviews and Meta-Analyses extension for Scoping Reviews. ${ }^{17}$

\section{Patient and public involvement}

It was not possible to involve patients or the public in the design or in the completion of the view. Findings from the scoping review will be disseminated to national and international experts working in the area, and to patients and the public through reports in relevant publications.

\section{Stage 1: identifying the research question}

Research questions in scoping reviews are naturally broad as the point of these types of reviews is to summarise a range of evidence. Through consultation with the research team and the Management of Addiction in Primary Care team at the Irish College of General Practitioners (ICGP), the following research questions were identified: (1) How have virtual consultations been used to provide OAT and related psychosocial supports? (2) How effective are these approaches? These questions might be refined or expanded on as the process of conducting a scoping review is often iterative and reflexive.

\section{Stage 2: identifying relevant studies}

In collaboration with the ICGP librarian and the research team, a comprehensive search strategy was developed. The researchers have selected a range of databases to ensure a variety of literature that encompasses views from general practice, psychiatry and psychology, health service delivery, and other medical and social sciences.

The relevant literature will include peer-reviewed publications and grey literature available from the following databases: PubMed, EMBASE, the Cochrane Library, PsycInfo and OpenGrey. Researchers will also hand search the references of included studies. To be eligible for inclusion, studies will have to report on our study population which is any person receiving or providing remote OAT or related psychosocial support. Remote care will include phone, video and email consultations, and prescription management.

Our search will include literature published in English from 2010 until the present. Due to technology improvements over the last decade, studies published prior to 2010 will be excluded. The preliminary search completed by the librarian and researcher RH determined these criteria would be adequate, as telemedicine is a new tool that has not yet been popularised everywhere. All searches will be completed in collaboration between the research team and librarians at the ICGP.

The first step consisted of an initial search of two databases (PubMed and EMBASE) using standardised search terms adapted to their requirements. Based on these searches, search terms will be redefined for a more comprehensive search of all included databases. The final step involves the hand searching of literature that meets the inclusion criteria.

\section{Stage 3: selection criteria}

The screening process will consist of two stages: a title and abstract review and full-text screening. Two reviewers will independently screen studies by title and abstract to determine suitability for inclusion. To be eligible for inclusion studies will have to report on models of telemedicine used to deliver OAT and/ 
or related psychosocial supports and be published in English in 2010 or later. OAT will include methadone and buprenorphine. Studies not deemed eligible for inclusion (where both researchers agree) will be removed. All other studies will move to stage 2 where the full texts will be reviewed. Both researchers will independently review the full study text and select studies to be included in the final review. The full team will review those that are undetermined and will make a final decision on their inclusion/exclusion.

\section{Stage 4: charting the data}

A data collection instrument will be developed by the research team to extract study characteristics. Study characteristics to be extracted will include: title, author, publishing body, publication date, peer-reviewed or grey literature, country/countries involved, keywords used by studies, aims of the study, methodology, study population, type of intervention used, type of care setting, health professionals involved in the treatment, satisfaction with technologies used, key findings and recommendations from the studies. After a preliminary charting of the first few papers, the principal investigator (DC) and research officer (RH) will review the results and make any changes to the characteristics being collected as required.

The data we extract from relevant studies will be charted and sorted into key themes using a qualitative thematic analysis approach. Quantitative data such as study population size will also be recorded alongside the qualitative information. We will pay particular attention to the types of telemedical interventions used-such as telephone, email or video consultation-and how effective both healthcare professionals and patients found them. After identifying themes from the literature, evidence will be synthesised using summary tables with key themes as headings as is common with scoping reviews.

\section{Stage 5: collating, summarising and reporting the results}

To increase the rigour of scoping review methodology, Levac et al have suggested that this section is divided into the following three separate steps: analysing the data, reporting results and applying meaning to the results. ${ }^{16}$ Our analysis will include both a descriptive numeric summary and a thematic analysis. This shall include characteristics of studies included, overall numbers of studies, study populations and where studies occurred. Qualitative analysis techniques will be used to complete the thematic analysis of this scoping review. Additionally, we will identify and report gaps in the available literature. Finally, we will consider the implications of the review's findings within a broader research, practice and policy context.

\section{Ethics and dissemination}

A scoping review is a secondary analysis of published literature and does not require ethics approval. This work will constitute the first part of developing a national model of remote OAT provision in Ireland so as to remove treatment barriers, particular in regional towns and rural areas, and also to inform how best to manage COVID-19-related risk for patients and healthcare providers. The results will be disseminated through local and national addiction expert groups, peer-reviewed publication and conference presentations.

Contributors $\mathrm{DC}$ and $\mathrm{RH}$-design of the protocol. $\mathrm{DC}$ and $\mathrm{RH}$-draft of the manuscript. DC, RH and ID-final approval of the manuscript.

Funding The authors have not declared a specific grant for this research from any funding agency in the public, commercial or not-for-profit sectors.

Competing interests None declared.

Patient and public involvement Patients and/or the public were not involved in the design, or conduct, or reporting, or dissemination plans of this research.

Patient consent for publication Not required.

Provenance and peer review Not commissioned; externally peer reviewed.

Open access This is an open access article distributed in accordance with the Creative Commons Attribution Non Commercial (CC BY-NC 4.0) license, which permits others to distribute, remix, adapt, build upon this work non-commercially, and license their derivative works on different terms, provided the original work is properly cited, appropriate credit is given, any changes made indicated, and the use is non-commercial. See: http://creativecommons.org/licenses/by-nc/4.0/.

\section{ORCID iDs}

Des Crowley http://orcid.org/0000-0002-8491-9596

Robyn Homeniuk http://orcid.org/0000-0002-5526-4113

\section{REFERENCES}

1 Thornton J. Clinicians are leading service reconfiguration to cope with covid-19. BMJ 2020;369:m1444.

2 Greenhalgh T, Koh GCH, Car J. Covid-19: a remote assessment in primary care. BMJ 2020;368:m1182.

3 Kirby T. Rheumatologists rapidly adjust patient care during COVID-19 pandemic. Lancet Rheumatol 2020;2:e258.

4 DegenhardtL, CharlsonF, FerrariA, et al. The global burden of disease attributable to alcohol and drug use in 195 countries and territories, 1990-2016: a systematic analysis for the global burden of disease study 2016. Lancet Psychiatry 2018;5:987-1012.

5 Degenhardt L, Peacock A, Colledge S, et al. Global prevalence of injecting drug use and sociodemographic characteristics and prevalence of HIV, HBV, and HCV in people who inject drugs: a multistage systematic review. Lancet Glob Health 2017;5:e1192-207.

6 Degenhardt L, Charlson F, Stanaway J, et al. Estimating the burden of disease attributable to injecting drug use as a risk factor for HIV, hepatitis $\mathrm{C}$, and hepatitis $\mathrm{B}$ : findings from the global burden of disease study 2013. Lancet Infect Dis 2016;16:1385-98.

7 Sordo L, Barrio G, Bravo MJ, et al. Mortality risk during and after opioid substitution treatment: systematic review and meta-analysis of cohort studies. BMJ 2017;357:j1550.

8 Cousins G, Boland F, Barry J, et al. J-shaped relationship between supervised methadone consumption and retention in methadone maintenance treatment (MMT) in primary care: national cohort study. Drug Alcohol Depend 2017;173:126-31.

9 Larney S, Peacock A, Leung J, et al. Global, regional, and countrylevel coverage of interventions to prevent and manage HIV and hepatitis $C$ among people who inject drugs: a systematic review. Lancet Glob Health 2017;5:e1208-20.

10 Sun Y, Bao Y, Kosten T, et al. Editorial: challenges to opioid use disorders during COVID-19. Am J Addict 2020;29:174-5.

11 Eibl JK, Gauthier G, Pellegrini D, et al. The effectiveness of telemedicine-delivered opioid agonist therapy in a supervised clinical setting. Drug Alcohol Depend 2017;176:133-8.

12 Marciano S, Haddad L, Plazzotta F, et al. Implementation of the $\mathrm{ECHO}^{\circledR}$ telementoring model for the treatment of patients with hepatitis C. J Med Virol 2017;89:660-4.

13 Lin LA, Casteel D, Shigekawa E, et al. Telemedicine-delivered treatment interventions for substance use disorders: a systematic review. J Subst Abuse Treat 2019;101:38-49. 
14 Zhou X, Snoswell CL, Harding LE, et al. The role of telehealth in reducing the mental health burden from COVID-19. Telemed J E Health 2020;26:377-9.

15 Arksey H, O'Malley L. Scoping studies: towards a methodological framework. Int J Soc Res Methodol 2005;8:19-32.
16 Levac D, Colquhoun H, O'Brien KK. Scoping studies: advancing the methodology. Implement Sci 2010;5:69.

17 Tricco AC, Lillie E, Zarin W, et al. PRISMA extension for scoping reviews (PRISMA-ScR): checklist and explanation. Ann Intern Med 2018;169:467-73. 